Voix et Images

volxetimages

\title{
LA CONSTITUTION DU LITTÉRAIRE EN CONTEXTE MÉDIATIQUE
}

La critique littéraire québécoise, 1860-1900

\section{THE CONSTITUTION OF A LITERARY SPACE IN A MEDIA} CONTEXT QUÉBEC LITERARY CRITICISM, 1860-1900

\section{LA CONSTITUCIÓN DE LO LITERARIO EN UN CONTEXTO MEDIÁTICO}

\author{
LA CRÍTICA LITERARIA QUEBEQUENSE, 1860-1900
}

\section{LOUIS-SERGE GILL}

Volume 42, numéro 3 (126), printemps-été 2017

Les genres médiatiques (1860-1900)

URI : https://id.erudit.org/iderudit/1041047ar

DOI : https://doi.org/10.7202/1041047ar

Aller au sommaire du numéro

\section{Éditeur(s)}

Université du Québec à Montréal

ISSN

0318-9201 (imprimé)

1705-933X (numérique)

Découvrir la revue

Citer cet article

GILL, L.-S. (2017). LA CONSTITUTION DU LITTÉRAIRE

EN CONTEXTE MÉDIATIQUE : la critique littéraire québécoise, 1860-1900. Voix

et Images, 42(3), 53-69. https://doi.org/10.7202/1041047ar
Résumé de l'article

La critique littéraire québécoise du XIX ${ }^{\mathrm{e}}$ siècle demeure l'un des genres médiatiques les plus délaissés des dernières années. Pourtant, nous observons dans la seconde moitié du siècle une croissance de la publication de textes de critique et un intérêt marqué pour la constitution d'instances de légitimation du littéraire. Dans cet article, l'auteur esquisse les formes et les tendances de la critique en contexte médiatique. À partir d'exemples tirés de journaux et de périodiques, il expose les formes suivantes : le prospectus, la bibliographie, l'article, l'étude, la correspondance et la biographie, avant de présenter le dossier de presse du Pèlerin de Sainte-Anne (1877), roman de Pamphile Le May, en regard du dialogue quotidien que permet la critique littéraire en contexte médiatique. 


\title{
LA CONSTITUTION DU LITTÉRAIRE EN CONTEXTE MÉDIATIQUE
}

La critique littéraire québécoise, $1860-1900$

$+\quad+$

LOUIS-SERGE GILL

Université du Québec à Trois-Rivières

\begin{abstract}
Et le pauvre lecteur, guidé par un tel écrivain, se promène tout un long article au milieu des potins et de toutes les animosités professionnelles. De grâce, peut-on appeler cela de l'appréciation ou de la critique! Quel bien peut-il bonnement résulter de pareils écrits ${ }^{1}$ ?
\end{abstract}

La critique d'avant 1900 demeure sans doute l'un des genres médiatiques et littéraires les plus délaissés des dernières années dans les études littéraires. Pourtant, des efforts individuels et collectifs ont déjà permis de localiser, d'inventorier et de décrire l'imposant corpus de réception d'avant les fameux essais de Charles Ab der Halden et de $\mathrm{M}^{\mathrm{gr}}$ Camille Roy. Si l'on se réfère à la bibliographie de David M. Hayne et Marcel Tirol$^{2}$ et à celle de René Dionne et Pierre Cantin ${ }^{3}$, aux analyses du Dictionnaire des œuvres littéraires du Québec ${ }^{4}$ ainsi qu'aux synthèses du collectif La vie littéraire au Québec ${ }^{5}$, sans oublier le numéro de Revue d'histoire littéraire du Québec et du Canada

1 Placide, «L'éloge et la critique», L'Art musical, vol. III, n 3, décembre 1898, p. 29. Nous n'avons pu retracer l'identité derrière ce pseudonyme. Malgré les similitudes avec Placide Lépine, l'auteur des «Silhouettes biographiques» dans L'Opinion publique en 1872 et pseudonyme collectif d'Henri-Raymond Casgrain et de Joseph Marmette, il est peu probable que Casgrain ait repris le «flambeau» après le décès de Marmette en 1895.

2 David M. Hayne et Marcel Tirol, Bibliographie critique du roman canadien-français, 1837-1900, Québec, Presses de l'Université Laval, 1969, 144 p.

3 René Dionne et Pierre Cantin, Bibliographie de la critique de la littérature québécoise et canadienne-française dans les revues canadiennes, t. III : 1760-1899, Ottawa, Presses de l'Université d'Ottawa, coll. «Histoire littéraire du Québec et du Canada français», 1992, 308 p.

4 Maurice Lemire (dir.), Dictionnaire des œuvres littéraires du Québec, t. I: Des origines à 1900, Montréal, Fides, 1978, 918 p. Dans chacune des notices, la réception est analysée, et on trouve en bibliographie les références du dossier de presse.

5 Les tomes I à V couvrent la période 1764-1918: Maurice Lemire (dir.), La vie littéraire au Québec, t. I: 1764-1805. La voix française des nouveaux sujets britanniques, Québec, Presses de l'Université Laval, 1990, 500 p.; Maurice Lemire (dir.), La vie littéraire au Québec, t. II: 1806-1839. Le projet national des Canadiens, Québec, Presses de l’Université Laval, 1992, 587 p.; Maurice Lemire et Denis Saint-Jacques (dir.), La vie littéraire au Québec, t. III: 1840-1869. Un peuple sans histoire ni littérature, Québec, Presses de l'Université Laval, 1996, 671 p.; Maurice Lemire et Denis Saint-Jacques (dir.), La vie littéraire au Québec, t. IV: 18701894. Je me souviens, Québec, Presses de l'Université Laval, 1999, 669 p.; Maurice Lemire et Denis SaintJacques (dir.), La vie littéraire au Québec, t. V: 1895-1918. Sois fidèle à ta Laurentie, Québec, Presses de l’Université Laval, 2005, 680 p. 
français (été-automne 1987) sous la direction de David M. Hayne consacré au sujet ${ }^{6}$, les textes de critique littéraire pullulent durant ce siècle mouvementé. Plus récemment, en 2011, Micheline Cambron a proposé une étude sur la réception entourant le Jean Rivard d'Antoine Gérin-Lajoie depuis $1837^{7}$, qui révèle un important corpus critique. Celui-ci se retrouve dans les préfaces d'œuvres, de recueils et d'anthologies, mais aussi au détour d'un vers ou d'une scène de roman. Il se dévoile plus précisément dans les journaux et dans les périodiques. Au fil des diverses publications, spécialisées ou non en littérature, le lecteur découvre une vaste bibliothèque d'œuvres et d'études. Force est de constater que, peu à peu, le XIx siècle s'est intéressé presque quotidiennement à la production littéraire, lui faisant côtoyer l'actualité politique, les faits divers et les développements scientifiques.

Dans cet article, nous souhaitons avant tout dégager les formes et les tendances de la critique littéraire en contexte médiatique. Reconnaître et distinguer la multiplicité des formes qu'elle y emprunte constitue une manière d'observer l'évolution du littéraire au Québec au xIx siècle. Dans les journaux quotidiens et hebdomadaires, elle épouse tantôt des formes brèves, tantôt des formes plus longues en fonction de la place que la ligne éditoriale accorde à la littérature. Dans les revues et périodiques, elle figure souvent à titre d'étude plus étoffée où se remarquent les liens entre le passé et le présent, l'ici et l'ailleurs.

Nous proposerons d'abord une typologie des formes de la critique présente dans les journaux et dans les périodiques. Sans que ce classement soit définitif, nous retiendrons des formes récurrentes, propres au contexte médiatique québécois entre 1860 et 1900 : le prospectus, la bibliographie, l'article, l'étude, et plus brièvement, la biographie, puis la «lettre». En nous inspirant des travaux de Daniel Chartier8, nous montrerons la façon dont ces formes cohabitent dans une réception au «quotidien», à partir du dossier de presse du Pèlerin de Sainte-Anne ${ }^{9}$ de Pamphile Le May.

Nous avons partiellement dépouillé, dans le cadre de notre thèse ${ }^{10}$, des journaux comme L'Opinion publique et Le Monde illustré, des périodiques comme les Soirées canadiennes, Le Foyer canadien et la Revue canadienne, pour constater que l'analyse littéraire se manifeste suivant diverses formes que l'on distingue soit par la rubrique qu'elle occupe (le prospectus et la bibliographie ${ }^{11}$ ), soit par son insertion

6 À propos de la littérature du xixe siècle, nous trouvons dans ce dossier des articles de Gilles Marcotte ("Octave Crémazie lecteur", Revue d'histoire littéraire du Québec et du Canada français, n 14, été-automne 1987, p. 15-27), de Francis Parmentier ("Arthur Buies et la critique littéraire», ibid., p. 29-35) et de Manon Brunet («L'historien Edmond Lareau et la critique littéraire au xIXe siècle», ibid., p. 37-57).

7 Micheline Cambron, «Lecture et non-lecture de Jean Rivard d'Antoine Gérin-Lajoie», Karine Cellard et Martine-Emmanuelle Lapointe (dir.), Transmission et héritages de la littérature québécoise, Montréal, Presses de l'Université de Montréal, coll. «Espace littéraire», 2011, p. 113-141.

8 Daniel Chartier, L'émergence des classiques. La réception de la littérature québécoise des années 1930, Montréal, Fides, coll. «Nouvelles études québécoises», 2000, 307 p.

9 Pamphile Le May, Le pèlerin de Sainte-Anne, Québec, Typographie de C. Darveau, 1877, 2 vol.

10 Louis-Serge Gill, Représentations privées et publiques de la figure d'écrivain au Québec, 1860-1900, thèse de doctorat, Trois-Rivières, Université du Québec à Trois-Rivières, en rédaction.

11 Nous faisons référence au titre donné à des colonnes de journaux ou à des sections de périodiques où l'auteur compile les nouvelles parutions d'ouvrages et d'autres imprimés. 
dans les genres journalistiques comme l'entrefilet ou l'étude consacrée à un sujet particulier, la biographie et la lettre, tous genres représentatifs de la critique littéraire en contexte médiatique. Bien qu'essentiellement théoriques, nos distinctions entre ces formes ont pour but d'ouvrir à une étude approfondie des manifestations du jugement littéraire avant 1900.

\title{
LA CRITIQUE LITTÉRAIRE ET SES FORMES EN CONTEXTE MÉDIATIQUE AU QUÉBEC, 1860-1900: ÉTAT DE LA RECHERCHE ET MÉTHODOLOGIE
}

Notre recherche tient compte des travaux déjà réalisés sur la critique littéraire en contexte médiatique. Comme nous l'évoquions en introduction, divers travaux de dépouillement et de repérage de ces textes ont été menés depuis les années 1970. Notamment, l'équipe du premier tome du Dictionnaire des œuvres littéraires du Québec propose des dossiers de presse pour chacune des œuvres traitées, facilitant ainsi leur repérage ultérieur par les chercheurs. Il en va de même pour l'imposant travail bibliographique exécuté par David M. Hayne et Marcel Tirol, puis par René Dionne et Pierre Cantin. Toutefois, il faut attendre L'institution du littéraire de Lucie Robert pour que s'esquisse une compréhension de la dynamique entre les commentaires et les œuvres, et plus précisément une reconnaissance du rôle du contexte médiatique dans le processus de réception:

\begin{abstract}
Cette première forme de critique qui apparaît dans les journaux se présente comme une sorte de spectacle de la littérature: un livre, une pièce sont des nouvelles au même titre que les autres dans un journal où la politique partisane tient lieu de politique éditoriale. Un livre, une pièce sont aussi des objets de consommation dont on fait la promotion par voie éditoriale: la critique est la première forme qu'emprunte la publicité ${ }^{12}$.
\end{abstract}

Si la critique littéraire des XvIII et xIX ${ }^{e}$ siècles se développe surtout dans une volonté de publiciser les œuvres, nous pouvons constater qu'elle occupe néanmoins une place de plus en plus grande dans les journaux et les revues. Par exemple, Kenneth Landry, dans ses travaux sur la diffusion de la littérature au milieu du $\mathrm{XIX}^{\mathrm{e}}$ siècle ${ }^{13}$, constate que les prospectus des Soirées canadiennes (1861) et du Foyer canadien (1863) proposent une vision de la littérature qui se développe en suivant des critères précis, principalement la conservation de la mémoire littéraire et populaire des Canadiens ${ }^{14}$.

12 Lucie Robert, L'institution du littéraire au Québec, Québec, Presses de l'Université Laval, coll. "Vie des lettres québécoises/Centre de recherche en littérature québécoise», 1989, p. 119.

13 Kenneth Landry, «La diffusion de la littérature au Québec vers le milieu du xix siècle. Le rôle des recueils littéraires (miscellanées et albums)", Aurélien Boivin, Gilles Dorion et Kenneth Landry (dir.), Questions d'histoire littéraire. Mélanges offerts à Maurice Lemire, Québec, Nuit blanche éditeur, coll. "Littérature(s)", 1996, p. 45-58.

14 Ibid., p. 56 . 
Après avoir distingué les particularités et les similitudes de chacune de ces manifestations de la critique, nous verrons brièvement, à partir du cas du Pèlerin de Sainte-Anne de Pamphile Le May, comment ces genres fonctionnent en système de réception, en nous appuyant sur les travaux de Daniel Chartier et de Micheline Cambron. Selon Chartier, «la publication de critiques à la parution d'une œuvre littéraire n'est pas un processus neutre d'accumulation des discours. C'est un mécanisme actif, au cours duquel une lutte se déroule conduisant à une interprétation dominante qui sera reprise par l'histoire littéraire ${ }^{15}$ ». Dans un article sur l'événement de lecture, Micheline Cambron, qui étudie les multiples réceptions de L'influence d'un livre (1837) de Philippe Aubert de Gaspé fils, suggère que la première réception fait écho à la publication du livre, la seconde à son insertion dans l'histoire littéraire et la troisième à son entrée dans le régime de la science ${ }^{16}$. Tout au long de notre article, nous en restons à cette première réception de l'œuvre, depuis la publicité jusqu'aux discours favorables et défavorables qui entourent sa parution.

\section{LE PROSPECTUS DE PÉRIODIQUE}

Généralement, le premier numéro d'un journal ou d'un périodique s'ouvre sur le prospectus, un texte de quelques colonnes ou de quelques pages où sont énumérés et expliqués les principaux objectifs de la publication. Ainsi, on y indique si la littérature et la réception des œuvres entreront dans les préoccupations des rédacteurs. Par exemple, en 1863, le comité éditorial du Foyer canadien met en évidence la question de l'accessibilité de la critique, puisque la lecture, et la discussion qui l'entoure, sont conçues comme essentiellement privées:

Il existe au milieu de nous des hommes instruits, éclairés, cultivant avec amour,
dans le silence de la retraite, la littérature et les sciences. Nous espérons qu'en leur
ouvrant ses pages, notre recueil pourra fournir à ces hommes modestes l'occasion
d'utiliser leurs loisirs en même temps que ceux des lecteurs, - qu'il rapprochera
tous ces amis des lettres, dont les voix isolées restent aujourd'hui sans écho, - qu'il
sera enfin comme le foyer où se réuniront toutes les intelligences du pays pour
échanger leurs vues, s'animer au contact les unes des autres, et s'entretenir un ins-
tant avec la grande famille canadienne ${ }^{17}$.

Le partage du savoir et son analyse n'appartiennent pas encore au monde médiatique. Mais presque au même moment, la préoccupation en faveur d'une pratique publique - et non plus uniquement privée - de la critique littéraire est énoncée dans le programme de la Revue canadienne:

15 Daniel Chartier, La réception de la littérature québécoise des années 1930, Montréal, Fides, coll. «Nouvelles études québécoises», 2000, p. 29.

16 Micheline Cambron, «De la critique comme événement ou le récit de la geste critique», Micheline Cambron et Gérard Langlade (dir.), L'événement de lecture, Québec, Nota bene, 2015, p. 173.

17 La rédaction, «Prospectus», Le Foyer canadien, t. I, janvier-février 1863, p. 8. Les auteurs soulignent. 
Par un choix varié de feuilletons, d'articles bibliographiques, et d'extraits ou de traductions en tout genre, le journal sera mis à la portée de tous les goûts, et rien ne sera épargné pour le rendre utile et agréable.

Le but de la Revue est moral, littéraire et national; nous ne négligerons aucun moyen de l'atteindre, et c'est pour nous aider, c'est pour réaliser ce dessein avec nous, que nous demandons le concours et l'encouragement de tous les Canadiens, sans distinction de partis, de localité ou d'opinions. La tâche que nous entreprenons est ingrate, difficile et pleine d'écueils; plusieurs ne la comprendront pas; mais elle nous sera rendue possible par de sages conseils et par une coopération active $^{18}$.

Cette revue «à la portée de tous les goûts» mise sur une solide équipe de collaborateurs pour discuter de littérature et poser sur elle un regard critique.

Quelque dix années plus tard, ce partage des œuvres correspond au mandat de L'Opinion publique, premier journal illustré au pays. Par une présentation matérielle plus conviviale que celle de la Revue canadienne, la rédaction veut faire entrer hebdomadairement les événements littéraires dans les foyers. C'est ainsi que, dès le prospectus, nous apprenons avec quelle rigueur les œuvres littéraires retenues pour publication sont choisies: «Notre littérature, nos feuilletons seront sévèrement choisis, et en partie l'œuvre d'écrivains canadiens. Nous n'oublierons jamais que le journalisme est un sacerdoce et qu'il faut non seulement instruire, plaire, mais encore et par-dessus tout, rendre meilleur ${ }^{19}$.» Le choix des œuvres dans L'Opinion publique et celui des collaborateurs dans la Revue canadienne témoignent de l'importance du cadre moral, et non littéraire, dans lequel s'élabore la critique.

Certains rédacteurs, comme Jules-Paul Tardivel, fondateur de La Vérité en 1881, exposent dès le prospectus leur méfiance à l'égard des instances «officielles» en matière de littérature. Ainsi, le 14 juillet 1881, Tardivel informe ses lecteurs de la création d'une Académie canadienne ${ }^{20}$ :

Si, d'un autre côté, négligeant les affaires matérielles, elle s'occupe exclusivement des choses intellectuelles, nous sommes prêt à parier dix contre un qu'elle sera tout bonnement une société d'admiration mutuelle, constituée légalement et organisée sur une vaste échelle. C'est-à-dire qu'elle sera un véritable fléau pour le pays et un grand obstacle aux progrès de la bonne et saine littérature ${ }^{21}$.

D'emblée, Tardivel diffuse dans son journal un discours anti-institutionnel ou, à tout le moins, neutre et éloigné des amitiés et des «admirations mutuelles ${ }^{22}$ ».

18 [La rédaction], «Prospectus», Revue canadienne, t. I, 1864, p. 5.

19 George E. Desbarats, J. A. Mousseau et L[aurent-]O[livier] David, "Au public», L'Opinion publique, vol. I, $\mathrm{n}^{\circ} 1,1^{\text {er }}$ janvier 1870, p. 1.

20 Vraisemblablement, il s'agit de la Société royale du Canada, fondée en 1882.

21 [Jules-Paul Tardivel], «L'Académie canadienne», La Vérité, vol. I, nº 1, 14 juillet 1881, p. 4.

22 Quelques années plus tôt, Arthur Buies adressait aussi des critiques virulentes à propos des coteries littéraires et des sociétés d'admiration mutuelle, principalement dans son prologue aux Petites chroniques pour 1877 (Québec, Imprimerie de C. Darveau, 1878, p. v-xxxvi). 
À la fin de la décennie 1880-1889, après une augmentation du nombre des publications littéraires et des activités éditoriales, le prospectus du Canada français, revue mensuelle publiée sous la direction d'un comité de professeurs de l'Université Laval, départage le bon grain de l'ivraie avant même que soit publiée une seule œuvre littéraire au sein de la revue: «Aucun travail ne pourra être admis s'il n'est excellent pour le fond comme pour la forme, c'est-à-dire que non seulement il devra être bien écrit et ne rien contenir de contraire à la foi catholique ou à la morale, mais qu'il devra, dans tous les cas, être un travail sérieux ${ }^{23}$.» À mi-chemin entre la critique privée et la critique professionnelle menée dans le cadre d'activités universitaires, ce programme très orthodoxe diffère grandement de l'ouverture démontrée dans les diverses publications de «jeunes» du début des années $1890^{24}$. En effet, dans le prospectus du Recueil littéraire, Pierre Bédard écrit:

Cependant depuis quelques années un mouvement plein de bon augure pour les lettres et les arts se produit au Canada; les journaux, les revues et les livres naissent et se répandent, les sociétés littéraires grandissent et les écrivains reçoivent enfin l'encouragement dû à leurs talents et à leur travail. Malgré quelques imperfections, entre autres celle de se laisser trop dominer par la puissance politique, les journaux canadiens d'aujourd'hui parlent un langage plus correct et se corrigent mutuellement de ces nombreux anglicismes dont ils abondaient; la génération des jeunes écrivains est d'une ardeur et d'un courage qui font présager aux lettres canadiennes un avenir brillant, et comme nous disait l'éminent économiste Claudio Jannet dans une de ses lettres: «Il y a encore de beaux jours pour la littérature française sur les rives du Saint-Laurent. $»^{25}$

Bédard revient sur l'ensemble des développements littéraires des décennies précédentes en soulignant, par exemple, l'existence de "sociétés littéraires» qui participent à l'encouragement des écrivains, tout en insistant sur la présence de jeunes lettrés talentueux de plus en plus actifs dans le champ littéraire.

Si la forme du prospectus varie peu au fil du siècle, l'intérêt pour la littérature et sa critique s'énonce de plus en plus clairement. Les auteurs des prospectus proposent un angle interprétatif lorsqu'ils s'affairent à délimiter la portée littéraire d'une publication. En retracer l'histoire complète tout au long du XIXe siècle nous confirmerait sûrement l'importance accordée à la littérature comme «savoir», ou encore au roman ou à la poésie comme genres légitimes.

23 L'administration, «Prospectus», Le Canada français, vol. I, nº 1, 1888, p. 6. Les auteurs soulignent.

24 Voir, notamment à propos de $L^{\prime} E$ Echo des jeunes, l'article de Michel Pierssens et Roberto Benardi, «L'Écho des jeunes: une avant-garde inachevée», Études françaises, vol. XXXII, n 3, automne 1996, p. 21-50.

25 Pierre Bédard, «Notre programme», Le Recueil littéraire, t. I, nº 1, 10 avril 1891, p. 1. 


\section{LA «BIBLIOGRAPHIE »}

La «bibliographie», que nous identifions par le titre sous lequel elle se présente tant dans les journaux que dans les périodiques, demeure, selon nos observations, la forme médiatique la plus courante. Elle rend compte du littéraire dans son principal médium: l'imprimé. Brève, elle s'insère aisément sur la page du journal, et fait une ou deux colonnes tout au plus. Dans les revues, notamment dans la Revue canadienne, elle s'étend généralement sur quelques pages. Qu'elle traite d'un ou de quelques ouvrages, de journaux ou de revues, elle suit un plan assez convenu: référence complète de l'ouvrage, présentation de l'auteur et, parfois, explication des conditions de production, suivies de quelques remarques, souvent non argumentées, sur le style et la valeur de l'ouvrage présenté dans un contexte plus large. Par exemple, dans L'Événement du 7 septembre 1876, Hector Fabre signe une recension du recueil posthume d'Elzéar Labelle, Mes rimes (1876), sans omettre les circonstances d'édition:

Le joyeux poète dont les poésies légères composent ce volume est mort il y a un an à peine. Il a laissé dans la mémoire de ses amis un renom d'esprit et de gaîté que ce volume ne fera que confirmer et qu'agrandir. À ces vers si lestement troussés, à cette verve toute gauloise, il est impossible de ne pas reconnaître une nature de satyrique et de chansonnier. Le bagage est léger, mais il suffit pour montrer toutes les qualités natives, originales, qui, dans un milieu plus favorable aux Muses, se fussent développées davantage et eussent atteint leur entier épanouissement ${ }^{26}$.

Après cet éloge posthume de l'auteur, Fabre consacre la majeure partie de sa recension au travail éditorial d'André-Napoléon Montpetit. Dans ce cas, l'espace laissé à la bibliographie ne permet pas de citer l'œuvre.

Dans La Minerve du 22 juillet 1882, Joseph Desrosiers signe une bibliographie plus substantielle des Échos (1882) d'Adolphe-Basile Routhier. La mise en contexte de l'ouvrage se présente comme suit:

M. le juge Routhier semble vouloir nous donner la mesure de son talent aussi bien que de son activité. À peine avons-nous eu le temps d'applaudir à la publication de son bel ouvrage "Ā travers l'Europe» qu'il nous présente un nouveau volume: des vers, cette fois. M. Routhier est déjà connu avantageusement comme poète, un grand nombre de ses productions ayant été publiées dans les journaux. Il réunit aujourd'hui ces morceaux épars, y ajoute des pièces inédites, et en fait un tout coordonné et homogène qu'il publie sous ce titre: Les échos, en le faisant précéder d'un [sic] introduction sur la poétique chrétienne ${ }^{27}$.

26 Hector Fabre, «Bibliographie: Mes rimes, par Elzéar Labelle, précédé d’une préface par A. N. Montpetit, P. G. Delisle, imprimeur", L'Événement, 7 septembre 1876, p. 2.

27 J[oseph] Desrosiers, «Bibliographie: Les échos d'Adolphe-Basile Routhier», La Minerve, 22 juillet 1882, p. 2 . 
Desrosiers revient sur la notoriété de l'auteur et sur l'importance de son ouvrage précédent avant de se lancer dans l'étude de divers aspects de l'œuvre, notamment par des regroupements thématiques et des citations pour appuyer ses propos.

En fin de bibliographie, les recommandations faites à l'auteur témoignent d'une vision de la littérature à l'adresse du lecteur:

Plus de travail et d'application aurait sans doute rendu cette versification plus correcte, plus souple et plus harmonieuse. Mais on conçoit aussi que l'auteur, ne faisant pas un métier de la poésie, et dont les instants sont réclamés par des travaux bien plus sérieux et plus importants, n'ait pu donner à son œuvre poétique tout le temps et tout le soin qu'elle réclamait. Telle qu'elle est, cette œuvre est cependant de nature à augmenter encore la grande réputation de M. Routhier; elle plaira au public, et, ce qui entre bien plus dans les intentions de l'auteur, elle fera du bien ${ }^{28}$.

Dans ce texte, Desrosiers insiste davantage sur les talents de prosateur de Routhier que sur ses compétences de versificateur. Il semble aussi que le jugement final revienne au public, à qui cette œuvre, malgré ses défauts, plaira sans aucun doute, selon Desrosiers.

Cette forme brève de critique littéraire permet presque de retracer l'ensemble de la production littéraire contemporaine des journaux et des périodiques. Par exemple, les œuvres proposées par L'Opinion publique font aussi l'objet de commentaires dans la Revue canadienne ou L'Événement. Dans l'ensemble, les bibliographies assurent une première réception de l'œuvre et confirment qu'elle a bel et bien été lue. La principale fonction du genre réside dans le recensement des œuvres, mettant les lecteurs au fait des dernières publications. D'ailleurs, le nombre croissant de périodiques spécialisés en littérature et en histoire, comme la Revue canadienne, invite à une métamorphose de cette forme. De brève, elle devient plus ample et s'intéresse à des aspects divers d'une œuvre, comme nous le montrions avec Mes rimes d'Elzéar Labelle. Nous pensons qu'elle se développe d'ailleurs en concomitance avec un autre genre, davantage tourné vers l'événement et l'actualité littéraires: l'article.

\section{L'ARTICLE}

Plus substantiel que la bibliographie et plus fréquent que l'étude, l'article trouve surtout place dans les journaux quotidiens ou hebdomadaires. Essentiellement, c'est par l'entremise de tels textes que les critiques rendent compte de l'actualité littéraire, c'est-à-dire des publications ou des soirées de lecture. On y mêle contexte de production et de diffusion, citations de l'œuvre et réaction du public. En ce sens, l'article diffère de la bibliographie par sa souplesse dans le choix du sujet, comme c'est le cas pour le poème Crâne et cervelle d'Eudore Évanturel. Dans son compte rendu intitulé «Soirée théâtrale», Hector Fabre, pour L'Événement, partage les impressions suivantes le 15 octobre 1875 :

28 Ibid. 
Crâne et cervelle a été fort bien accueilli, et est un joli début. Le sujet est étrange et lugubre; il s'agit du vol d'un cadavre et la scène principale se passe dans une salle de dissection à l'école de médecine; la tombe violée renfermait le corps de la fiancée du carabin auteur du vol.

Il faut avouer que pareil sujet prête à des images fortes, sauvages, sinistres. Aussi les vers portent profondément l'empreinte du sujet: la pensée s'y traduit souvent avec originalité. Le style est châtié et les rimes heureuses ${ }^{29}$.

Bien que Fabre relève les qualités et les difficultés inhérentes au sujet du poème, il rappelle l'importance de la tâche qui attend Évanturel: «Nous l'engageons à ne pas négliger la muse; il a du talent, le feu sacré mais [...] il faut étudier et étudier sans relâche. C'est le conseil que nous nous permettons de donner à M. Évanturel. Il fera marque s'il le suit à la lettre ${ }^{30}$.» Dans l'article, le lecteur, ou le spectateur dans ce cas précis, se présente souvent comme le témoin des écueils inhérents à la vie littéraire, d'où les invitations nourries à poursuivre avec acharnement le travail, comme le faisait Desrosiers dans la recension bibliographique citée précédemment. Dans le même ordre d'idées, Jean-Baptiste Caouette commente, quelques années plus tard, les vers d'Évanturel publiés dans L'Opinion publique sous le pseudonyme de Talma. Pour l'occasion, il revient sur la «soirée théâtrale» et rédige un article similaire à celui de Fabre:

Pendant une quinzaine de jours, on ne parla, à Québec, que de Crâne et cervelle; et les journaux - toujours prodigieux de louanges - annoncèrent, en gros caractères, que M. Évanturel s'était décidé de livrer son poème à la publicité, mais qu'avant, il voulait qu'il fût solennellement déclamé au Music Hall. En apprenant cette nouvelle, tous les hommes de lettres - jusqu'à moi, chers lecteurs - brûlèrent du désir d'aller entendre, à la Salle de Musique, ce poème qui faisait tant de bruit ${ }^{31}$.

L'événement autour de Crâne et cervelle suscitait l'engouement et l'intêrêt des gens de lettres, haussant considérablement les attentes pour la suite, au plus grand désarroi d'Évanturel:

Mais comme les plus grands bonheurs en ce bas monde sont souvent de courte durée, quelques jours plus tard, un bon écrivain publia, dans le Journal de SaintRoch, une critique très sévère de Crâne et cervelle. Et, en lisant cette critique, le poète en fut soudainement frappé au cœur; les rêves brillants que son imagination avait formée [sic] disparurent au choc de la réalité.

Oui, je le répète, $M$. Évanturel est doué d'un bon talent, il possède une véritable nature de poète; mais ce qui lui manque, c'est cette énergie fébrile, cette persévérance dans le travail, qualités essentielles du vrai poète ${ }^{32}$.

\footnotetext{
29 [Hector Fabre], «Soirée thêâtrale», L'Événement, 15 octobre 1875, p. 2.

30 Ibid.

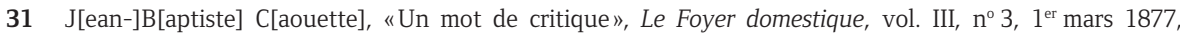
p. 163. L'auteur souligne.

32 Ibid., p. 164.
} 
L'article de critique littéraire se nourrit des événements littéraires, de leur actualité, mais aussi de leur pérennité dans la mémoire des gens. Avant l'étude plus substantielle de l'œuvre, ce condensé offre un avant-goût et une suite à la réception des productions d'un écrivain. Il permet de rendre compte à la fois de la part matérielle (livre, imprimé) et immatérielle (lecture en public, réception immédiate) de l'œuvre. Aussi, nous pourrions aisément rapprocher ce genre de la chronique et du reportage dans la mesure où se remarque dans ces deux pratiques la présence constante de l'énonciateur, que nous retrouvons, d'une certaine façon, dans la bibliographie, et plus encore dans le prospectus. $\mathrm{Si}$, parfois, la réception d'un texte s'arrête à la bibliographie, parfois les gens de lettres en font un événement littéraire dont on traite dans l'article. Hector Fabre, auteur de nombreux articles littéraires pour L'Événement, s'y adonne un peu comme à la chronique. C'est en quelque sorte l'expérience personnelle de l'événement littéraire (nous évoquions la représentation publique de Crâne et cervelle d'Évanturel) qui permet de rendre compte de l'expérience collective.

\section{L'ÉTUDE}

L'étude se distingue de l'article dans la mesure où elle tient sur plusieurs pages, voire sur plusieurs livraisons, et qu'elle se trouve surtout dans les périodiques et les revues $^{33}$. Cette forme présente une analyse en profondeur d'une question spécifique, d'une œuvre littéraire ou de la production d'un auteur. Les études sont souvent issues de conférences comme la "Causerie sur la littérature canadienne» d'Hector Fabre publiée dans les pages du Canadien les 31 mars $^{34}$ et 2 avril $1866^{35}$, ou encore celle de Faucher de Saint-Maurice, «L'homme de lettres: sa mission dans la société moderne ${ }^{36}$ ", publiée en 1868 dans La Revue canadienne. Mais peu à peu, des études originales, comme celle que Pamphile Le May propose dans la Revue de Montréal en $1877^{37}$, trouveront leur place dans les périodiques.

De manière générale, l'étude littêraire rend compte, exemples à l'appui, d'une problématique historique ou critique. Par exemple, Alphonse Lusignan signe en 1886, dans les Nouvelles soirées canadiennes, une étude intitulée «Nos premiers rapports littéraires avec la France ${ }^{38}$ ». Le texte suit un plan assez commun: présentation de fond de la question et histoire du phénomène jusqu'à ses plus contemporaines

33 Cependant, nous trouvons également de longues études dans certains journaux, comme celle qu'HenriRaymond Casgrain consacre à Angéline de Montbrun dans L'Opinion publique du 6 décembre 1883 (vol. XIX, $n^{\circ} 49$, p. 1-2). De manière exceptionnelle, cette étude occupe les deux premières pages du journal illustré, créant ainsi un événement autour de l'œuvre de la première romancière canadienne.

34 Hector Fabre, «Causerie sur la littérature canadienne [1/2]», Le Canadien, 35e année, nº 151, 31 mars 1866 , p. 1.

35 Hector Fabre, "Causerie sur la littérature canadienne [2/2]", Le Canadien, 36e année, n 1,2 avril 1866, p. 1.

36 Narcisse-Henri-Édouard Faucher de Saint-Maurice, «L'homme de lettres: sa mission dans la société moderne», Revue canadienne, t. V, 1868, p. 437-451.

37 Pamphile Le May, «Quelques poètes illettrés de Lotbinière», Revue de Montréal, t. 1, 1877, p. 53-64 et p. 89-96.

38 Alphonse Lusignan, Nouvelles Soirées canadiennes, vol. V, nº 10, octobre 1886, p. 433-446. 
manifestations. En offrant une place à l'ensemble des écrivains québécois ayant reçu des échos en France, il note, à propos de son ami Louis Fréchette: «Entre Canadiens, on est habitué à se déchirer. Je sais qu'il est de mode de se rabaisser entre hommes qui s'adressent au public dès que l'un d'eux a l'infection politique. On a cherché à dépercher Fréchette des hauts sommets qu'il a atteints. On a manqué son coup, je le dis avec plaisir ${ }^{39}$.» Si d'une part le développement s'appuie sur la rigueur et l'érudition dans les exemples donnés, la liberté d'opinion de la conclusion fait d'autre part de l'étude l'une des formes les plus utiles pour saisir l'évolution de la critique littéraire en contexte médiatique ${ }^{40}$. Il semble que les rédacteurs veuillent rendre compte à la fois des événements littéraires et de la matière intellectuelle et stylistique des œuvres, tout en offrant au lecteur une perspective argumentée sur les textes et sur les questions qui entourent leur production, leur diffusion et leur légitimation au Québec comme à l'étranger. De manière générale, en plus d'offrir aux écrivains une certaine visibilité, l'étude les situe les uns par rapport aux autres dans l'institution littéraire, et cela témoigne de l'étendue du marché auquel ils sont de plus en plus contraints de participer. L'étude s'apparente à la «seconde réception ${ }^{41} » \mathrm{~d}^{\prime}$ une œuvre. Forme amplifiée de la critique, elle explore l'inscription de l'œuvre dans une tradition esthétique et morale. On y examine le style, le contenu et, au besoin, on en résume l'intrigue et le récit. Telle qu'elle se pratiquait à l'époque, l'étude s'apparente aux nombreux essais de Camille Roy des premières décennies du xx siècle.

\section{LA BIOGRAPHIE}

Que ce soit dans les journaux ou dans les périodiques, le lecteur rencontre aussi des biographies et des portraits. Présents à des moments charnières dans la vie d'un écrivain (publications, entreprises diverses, couronnement littéraire ou décès), ces textes retracent le parcours des gens de lettres, en insistant la plupart du temps sur la grandeur de l'œuvre. Nous connaissons les fameuses «Silhouettes et pastels littéraires» (1872) de Placide Lépine (pseudonyme d'Henri-Raymond Casgrain et de Joseph Marmette), et au tournant des années 1890, dans Le Monde illustré, les «Galeries canadiennes» d'Édouard-Zotique Massicotte. Les travaux de Manon Brunet sur les «Silhouettes biographiques» de Placide Lépine, publiées dans L'Opinion publique en 1872, révèlent la conception de la littérature et de la critique entretenue par Casgrain et Marmette:

Dans cette perspective critique unidimensionnelle, car l'homme et l'œuvre forment un tout indivisible, les lignes de l'écriture sont celles de l'homme. Dès lors, il suffit de montrer l'homme et de deviner la forme et le sens de son écriture, sa valeur

39 Alphonse Lusignan, "Nos premiers rapports littéraires avec la France», Nouvelles Soirées canadiennes, vol. V, no 10, octobre 1886, p. 446.

40 Notons que, dans la seconde moitié du XIX $x^{e}$ siècle, il n'existe pas encore de critique universitaire.

41 Micheline Cambron, «De la critique comme événement ou le récit de la geste critique», Micheline Cambron et Gérard Langlade (dir.), L'événement de lecture, Québec, Nota bene, 2015, p. 173. 
esthétique. L'œuvre n'a pas plus d'indépendance d'esprit, d'autonomie que la critique qui en parle ${ }^{42}$.

Ces biographies, attachées à des personnalités politiques et littéraires, dressent en quelque sorte un bilan de fin de siècle. C'est ainsi que sous la plume de Massicotte prend enfin vie, entre autres, le poète, traducteur et érudit Alfred Garneau, «un de nos rares écrivains qui s'est rendu maître de la langue française ${ }^{43}{ }^{\prime}$. Cette présentation des écrivains, dans leurs occupations personnelles et professionnelles, est une forme suffisamment répandue pour qu'on rassemble en volume presque systématiquement les textes de certains piliers du genre, comme Louis-Michel Darveau ${ }^{44}$, Henri-Raymond Casgrain ${ }^{45}$ et Laurent-Olivier David ${ }^{46}$. Ces galeries de "personnages » s'opposent en somme aux événements quotidiens qu'évoque la lettre d'écrivain, forme plus spontanée d'appréciation littéraire.

\section{LA LETTRE D'ÉCRIVAIN}

Les lettres envoyées par les écrivains aux journaux et aux périodiques soulignent bien leur implication dans une démarche collective. Petit à petit, le littéraire s'inscrit dans des débats d'idées et s'intéresse à des questions politiques et religieuses. Cela peut d'ailleurs être rapporté à la recherche d'une autonomie littéraire caractéristique de cette période.

L'engagement des hommes de lettres dans les débats politiques et idéologiques place parfois les œuvres littéraires au premier plan des échanges et des débats. C'est ainsi que nous observons le développement d'une critique littéraire qui s'exprime à travers la correspondance et la lettre d'opinion publiées dans les périodiques. De fait, la prise de parole publique des écrivains sur des sujets qui débordent du contexte de leurs œuvres décloisonne les espaces où se manifeste habituellement la critique littéraire, entre autres par des échanges parfois véhéments qui en font une pratique risquée ou lourde de conséquences pour une carrière littéraire.

Genre médiatique typique des quotidiens et plus ancien que les formes relevées précédemment, la lettre constitue la majeure partie du corpus des Guêpes canadiennes éditées par Augustin Laperrière en 1882 et en 1884. Cette anthologie regroupe des textes essentiels de la critique idéologique et polémique au xix siècle: les Lettres à Basile (1871) de Louis Fréchette, tirées de L'Événement, et les Causeries du dimanche (1872) d'Adolphe-Basile Routhier, tirées du Courrier du Canada.

42 Manon Brunet, «"Silhouettes", "portraits" et "profils". La critique biographique de "nos hommes de lettres" au XIXe siècle», Annette Hayward et Agnès Whitfield (dir.), Critique et littérature québécoise. Critique de la littérature/littérature de la critique, Montréal, Triptyque, 1992, p. 104.

43 Édouard-Zotique Massicotte, "Galerie canadienne: M. Alfred Garneau», Le Monde illustré, ge année, $n^{\circ} 468,22$ avril 1893, p. 601.

44 Louis-Michel Darveau, Nos hommes de lettres, Montréal, Imprimé par A. A. Stevenson, 1873, 276 p.

45 Henri-Raymond Casgrain, Euvres complètes, t. II: Biographies canadiennes, Québec, Typographie de C. Darveau, 1875, $97 \mathrm{p}$.

46 Laurent-Olivier David, Biographies et portraits, Montréal, Beauchemin \& Valois, 1876, 301 p. 
Routhier commente non seulement l'idéologie libérale, mais aussi sa présence dans les œuvres littéraires de Fréchette, dont La voix d'un exilé. Il s'ensuit alors une vague de réponses: Buies envoie sa "Chronique québecquoise ${ }^{47}$ » et Louis-Antoine Dessaulles réplique à Routhier dans L'Événement ${ }^{48}$. La question soulevée par la lettre envoyée au journal est certainement celle du capital symbolique qui peut être acquis par l'entremise de luttes idéologiques bien connues de l'époque: les classiques et les modernes, les ultramontains et les libéraux. L'argumentation déployée dans ces lettres vise avant tout la victoire d'un système de pensée sur un autre. Bien qu'il en existe des exemples antérieurs à 1870, l'initiative de Laperrière d'éditer des recueils à deux voix témoigne de leur importance pour le développement de la critique, de l'histoire et même de l'institution littéraires. La lettre, en plus de témoigner du choc entre des idéologies et des perspectives esthétiques, place les écrivains au centre des débats de société par lesquels ils abordent ou non des questions littéraires. En ce sens, nous pouvons dire qu'elle fait de la critique un engagement de premier ordre pour certains, notamment lorsque leur réputation est en jeu. Qui ose critiquer sera critiqué en retour.

\section{LE LITTÉRAIRE AU «QUOTIDIEN»: LE CAS DU PÉLERIN DE SAINTE-ANNE (1877) DE PAMPHILE LE MAY}

Compte tenu de la présence croissante de la critique littéraire sous toutes ses formes à la fin des années 1870, le cas du roman Le pèlerin de Sainte-Anne de Pamphile Le May s'avère intéressant pour observer la première étape du processus de réception que nous évoquions en nous appuyant sur les travaux de Micheline Cambron et de Daniel Chartier. Le dossier de presse du roman rend compte d'une variété de sources ${ }^{49}$ et de points de vue. À partir de ces textes, nous pouvons voir comment les différentes formes de la critique littéraire cohabitent au quotidien (ou presque) pour en venir, en quelques mois, à clore le débat sur l'œuvre. Nous suivrons les étapes du processus de réception comme suit: recension et exposition de l'œuvre, critique sous diverses formes dans des journaux et des périodiques, et finalement repérage d'une synthèse qui recense, cite et analyse les réceptions antérieures, tout en formulant une opinion sur l'état de cette pratique ${ }^{50}$.

47 Arthur Buies, «Chronique québecquoise», Le Pays, 24 octobre 1871, p. 2.

48 Louis-Antoine Dessaulles, «Correspondances», L'Événement, 18 janvier et 5 février 1872, p. 2.

49 Le dépouillement de journaux et de périodiques déjā fait par Rémi Ferland dans son édition critique du Pèlerin de Sainte-Anne nous a servi à composer notre dossier de réception. Le lecteur trouvera l'entièreté de ce dossier à la fin de l'édition du roman (Pamphile Le May, Le pèlerin de Sainte-Anne, édition établie, présentée et annotée par Rémi Ferland, Sainte-Foy, Éditions de la Huit, coll. «Anciens», 1998, p. 375-399). Chacun des textes cités dans le présent article a été retracé et cité à partir de son contexte d'origine.

50 Comme le signale Daniel Chartier à propos du système de réception des années 1930, "[1]es critiques semblent d'abord aveugles les uns aux autres jusqu'au moment où un phénomène particulier, parfois un seul texte auquel ceux qui suivent feront systématiquement référence, détermine une trajectoire dans l'organisation du système de réception ». L'émergence des classiques : la réception de la littérature québécoise des années 1930, Montréal, Fides, 2000, p. 282. 
Le premier projet en prose de Pamphile Le May a bénéficié d'une certaine publicité. D'abord connu pour ses œuvres poétiques, dont deux poèmes couronnés dans le cadre du Concours de poésie de l'Université Laval, Le May s'inspire d'un fait divers comme matière pour ce premier roman à la trame tragique. Dès le 14 décembre 1876, Le May sollicite l'aide de souscripteurs par l'entremise de L'Opinion publique ${ }^{51}$ et bénéficie du soutien de l'Institut canadien de Québec, où se déroulera le premier événement autour de ce roman. De fait, Le Canadien et Le Journal de Québec font la publicité, le 11 avril 1877, d'une lecture publique de quelques extraits du roman par l'auteur. Le lendemain, le 12 avril, le critique du Canadien s'en prend fortement à la manière réaliste de ce «romantique de la plus belle eau [...], de la pire espèce» :

Notre romancier fait, en passant, une tirade contre notre système d'éducation et atteint, par ricochet, le gouvernement de la province. De nos jours cela semble de rigueur. C'est la mode de dire que les Canadiens sont le peuple le plus ignorant du monde. Heureusement, encore une fois, le livre de M. Le May n'ira pas à l'étranger. Somme toute, les chapitres du Pèlerin de Sainte-Anne que nous avons entendus renferment quelques beautés et beaucoup de défauts. Si nous avons particulièrement insisté sur ces derniers, c'est que, nous le savons d'avance, d'autres journaux vont rendre ample justice aux premières. À eux de flatter l'amour-propre de l'auteur, à nous de respecter la vérité ${ }^{52}$.

L'appréciation de l'autre partenaire de l'événement est publiée la même journée sous forme d'article et est plus clémente à l'endroit du romancier. Non seulement cette séance fut, selon l'auteur anonyme, l'une des meilleures données par l'Institut canadien de Québec, mais les spectateurs « ont eu un avant-goût de cette œuvre que nous n'hésitons pas à mettre au rang des meilleures qui aient été publiées dans ce pays, pour ne pas dire plus ${ }^{53}$ ». Deux jours plus tard, c'est au tour d'Hector Fabre de saluer l'œuvre de Le May dans un article de L'Événement:

Le pèlerin de Sainte-Anne est un roman essentiellement canadien: c'est la nature du pays, de ses habitants, les mœurs villageoises prises sur le vif, photographiées instantanément. Ce qui est mieux, c'est que, laissant de côté les traditions de la vieille école du coloris, le peintre de nos mœurs rurales est resté dans le vrai ${ }^{54}$.

Finalement, sur la même page, on ajoute une réponse du romancier à son détracteur du Canadien, qui laisse entendre qu'il connaît l'identité de celui-ci:

Quelqu'un m'a dit que vous vous promettez de me faire perdre cent souscripteurs, si je réplique à votre malicieux compte rendu. Pourquoi, monsieur, me feriez-vous ce mal? Est-ce que la loi divine, qui nous défend de nuire à notre prochain, ne descend

51 Ibid., p. 375-376 pour l'article et p. 399-409 pour la liste des souscripteurs.

52 [S. n.], «Le pèlerin de Sainte-Anne», Le Canadien, 12 avril 1877, p. 2.

53 [S. n.], «Fait divers», Le Journal de Québec, 12 avril 1877, p. 2.

54 [Hector Fabre], «Le pèlerin de Sainte-Anne», L'Événement, 14 avril 1877, p. 2. 
pas jusqu'à vous? Vous allez protester de votre franchise, mais votre premier article vous démasque ${ }^{55}$.

Un climat de menace s'instaure dès les premières recensions. On s'attaque à la moralité de l'auteur, qui riposte en remettant en question celle du critique caché derrière $l^{\prime}$ anonymat ${ }^{56}$. Ainsi, les articles et la lettre de l'auteur témoignent de vives discussions autour du projet romanesque de Le May.

Après cette première phase de réception de l'œuvre, il faut attendre juin 1877 pour que paraissent, respectivement dans le Journal de Québec et dans Le Nouveau Monde, deux articles sur l'ouvrage imprimé. Le premier article paraît sous la plume de Jules-Paul Tardivel le 11 juillet 1877 dans Le Canadien. Malgré quelques notes positives, Tardivel ne ménage rien pour abaisser le roman de Le May avec comme argument principal que son réalisme le rend immoral. Cet article, qui occupe la quasitotalité d'une page du quotidien, vilipende ceux des auteurs canadiens qui osent suivre les auteurs français à la mode. Ainsi, Le May fait les frais d'une comparaison avec Émile Zola :

M. Le May est tombé dans le réalisme. Je ne veux pas le comparer à Émile Zola, ni son livre à L'assommoir. Au moins le but de l'auteur canadien est bon; mais s'il ne descend pas aussi bas que l'écrivain français, c'est que notre jeune société n'est pas aussi gangrenée que la société du Vieux Monde ${ }^{57}$.

Deux jours plus tard, le Journal de Québec riposte en soulignant la mauvaise foi de Tardivel et en affirmant que «Le pèlerin de Sainte-Anne est un roman national fort bien conçu, et une peinture des mœurs d'une incontestable fidélité-58 ${ }^{5}$. Le 17 juillet, le même Journal publie à la fois la réponse de Tardivel ${ }^{59}$ et un bref article soulignant que l'on n'a pas voulu être injuste envers le commentateur ${ }^{60}$.

À ce stade nous pouvons déjà remarquer le jeu entre le discours sur l'œuvre et celui qui s'attache à sa réception, notamment par la citation et le commentaire. La synthèse critique s'illustre dans un article que Delta (pseudonyme de Benjamin Sulte) signe dans L'Opinion publique du 9 août 1877. Sulte ne saurait énoncer plus clairement ses intentions lorsqu'il revient d'entrée de jeu sur les diverses lectures, soit celles du Journal de Québec et du Nouveau Monde. Par l'entremise de citations et d'arguments, il cherche à départager les bonnes des moins bonnes et, inévitablement, il aborde le(s) texte(s) de Tardivel paru(s) dans Le Canadien. Médiateur et conciliateur, Sulte parvient à la conclusion suivante:

Si M. Tardivel avait terminé sa critique en disant qu'à part certaines pages où on trouve les défauts qu'il a signalés, le roman de M. Le May est généralement bien

55 Pamphile Le May, «Correspondance», L'Événement, 14 avril 1877, p. 2.

56 Il s'agit probablement de Jules-Paul Tardivel, qui récidivera - en signant cette fois - à la sortie du livre.

57 Jules-Paul Tardivel, «Le pèlerin de Sainte-Anne», Le Canadien, 11 juillet 1877, p. 2.

58 [S. n.], «Le pèlerin de Sainte-Anne», Le Journal de Québec, 13 juillet 1877, p. 2.

59 Jules-Paul Tardivel, «Le pèlerin de Sainte-Anne», Le Journal de Québec, 17 juillet 1877, p. 2.

60 [S. n.], [s. t.], Le Journal de Québec, 17 juillet 1877, p. 2. 
écrit et qu'il dénote chez l'auteur le talent, la force de conception et d'imagination nécessaires pour se signaler dans le roman, nous serions d'accord. Tous les jours on dit, en contemplant un édifice, un monument: «Il y a un défaut ici, il y a un défaut là; mais le plan, le dessin indiquent un homme de talent, un véritable architecte.» Ainsi, nous disons, en contemplant l'œuvre de M. Le May: il y a des imperfections dans cet ouvrage, mais il dénote chez l'auteur le talent qu'il faut pour obtenir des succès dans ce genre de littérature ${ }^{61}$.

De tous les écrits entourant l'événement et la publication du Pèlerin de Sainte-Anne, celui de Sulte est le seul qui appelle à une vision plus indulgente de la créativité et de l'inventivité. Dans cette synthèse des points de vue, plutôt que d'insister sur l'observation de références immorales par le romancier, Sulte souligne l'absence de modèles et de prédécesseurs dans le genre du roman réaliste au Canada. Plus largement, ce débat autour du réalisme atteint même les tenants de créations plus intimistes. En effet, le débat, loin d'être terminé, se poursuit en 1878 avec la parution des Premières poésies d'Eudore Évanturel. Tardivel et d'autres attaqueront autant le réalisme intimiste d'Évanturel que l'indulgence de son préfacier, Joseph Marmette. Ce dernier, sans se complaire dans l'admiration des vers d'Évanturel, met en évidence quelques faiblesses quant aux modèles littéraires et aux effets recherchés. De fait, Marmette tente même de signaler la filiation «probable» d'Évanturel au «poète national», Octave Crémazie:

Petit-fils du soldat de Napoléon, chanté par Crémazie, M. Évanturel a dû sentir passer autrefois sur son front le souffle inspiré de notre barde canadien. [...] Saisi d'une noble émulation, que le jeune poète accorde aussi sa lyre à l'unisson de la harpe de l'auteur du Drapeau de Carillon et qu'il entonne la mélopée des combats de nos aïeux ${ }^{62}$ !

Même pour le préfacier et premier critique de l'œuvre, il semble qu'il faille atténuer les élans intimistes et les incursions dans la peinture réaliste. Cela étant dit, si certains ont étouffé la voix intimiste d'Évanturel, les critiques acerbes des ultramontains n'auront pas empêché Pamphile Le May de publier deux autres romans de facture semblable.

Tout au long du xixe siècle, la critique littéraire se fait sous différents modes et s'attache à des problématiques tout aussi variées. Si nous jugions important de nous intéresser surtout à la période 1860-1900, c'est qu'à ce moment, la critique prend forme. Cette diversité (prospectus, bibliographie, article, étude, biographie et lettre)

61 Delta [Benjamin Sulte], «Le pèlerin de Sainte-Anne par M. Pamphile Le May», L'Opinion publique, vol. VIII, $\mathrm{n}^{\circ} 32,9$ août 1877, p. 374.

62 Joseph Marmette, «Préface», Eudore Évanturel, Premières poésies, 1876-1878, Québec, Augustin Côté et $\mathrm{c}^{\mathrm{ie}}$, p. 55. 
ne va pas de soi. Une histoire approfondie de l'apparition et du développement de ces formes serait nécessaire, mais nous avons voulu, dans la première partie de notre article, contribuer à les faire connaître en mettant en lumière leurs particularités et leurs caractéristiques. Dans la seconde partie, il s'agissait de montrer comment elles participent toutes à la réception d'une œuvre et culminent dans la formation d'un point de vue analytique plus élaboré tenant compte des divers textes écrits sur cette même œuvre.

Il ne faut pas oublier qu'à la fin du xIXe siècle, qu'ils s'immiscent dans les débats de leur époque ou qu'ils se fassent discrets, les critiques sont eux-mêmes des écrivains. Dans ce contexte, il importerait d'évaluer et d'étudier sérieusement la portée des textes rédigés par les auteurs en marge de leurs œuvres mieux connues. Les polémiques entourant le réalisme du Pèlerin de Sainte-Anne ou celles concernant l'intimisme d'Eudore Évanturel dans ses Premières poésies, et la réponse ou la réaction des auteurs visés, ne sont que quelques-uns des exemples de la constitution du littéraire comme savoir et système de référence par l'entremise de journaux et de périodiques. Mais la critique à elle seule contient des informations pour les chercheurs qui s'intéressent aux trajectoires d'écrivains, aux pratiques de lecture et, surtout, aux modalités suivant lesquelles on reçoit les œuvres littéraires au cours du siècle, que ce soit d'un point de vue esthétique ou idéologique. Au XIX siècle, la critique n'est pas faite que d'oppositions binaires. L'histoire de ses formes, de ses tendances et de ses écrivains nous permettrait de voir, entre autres, que les luttes incessantes qui jalonnent le xix siècle sont en fait, d'un point de vue littéraire, le moteur d'une inlassable conversation entre des individus passionnés par la création d'un savoir et engagés dans un processus de connaissance historique, esthétique et social. 